\title{
Opening the gates to molecular medicine
}

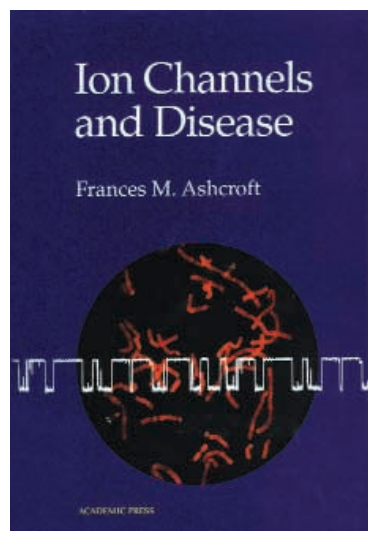

\author{
Ion Channels and Disease - \\ Channelopathies \\ by Frances Ashcroft \\ Academic Press · October 1999 \\ Hardback £46.95/\$75 \\ Blanche Schwappach
}

\begin{abstract}
$\mathrm{n}$ this age of constantly updated web pages relating the latest breakthroughs in biomedical research, who would attempt to write not just a review article but a whole book on a specialized area like 'channelopathies'? Frances Ashcroft from the University Laboratory of Physiology at Oxford did, and her colleagues in related fields, as well as advanced students interested in molecular medicine, are very fortunate that she embarked on this project. The term 'channelopathies', as defined by the author, "describes those human and animal diseases that result from defects in ion channel function". Like any problem in molecular medicine, understanding channelopathies requires an approach that integrates several layers of biological complexity - from gene sequence to protein function to cellular regulation to organ physiology. The recent explosion of sequence data available to the scientific community has accelerated certain steps in the corresponding research so much that keeping track has become a challenge. No wonder the author felt "like the Red Queen in Alice in Wonderland, who had to run ever faster in order to remain in the same place" while writing this book.
\end{abstract}

But why do ion channels warrant a special term for such an ostensibly common circumstance, in which malfunction of a protein can lead to disease? What special contribution does the ion channel field add to the general endeavour of molecular medicine? Searching OMIM, the catalogue of genetically linked diseases accessible via the website of the National Center for Biotechnology Information (http://www.ncbi.nlm.nih.gov.) tells us that it is not numbers. Although the search term 'channel ${ }^{*}$ yields an impressive number of entries (388, not necessarily all channelopathies), so do 'protease ${ }^{*}$ (314), 'kinase ${ }^{\star}$ ' $(1,143)$ and 'transcription factor $^{\star}$ ' $(1,019)$. The answer may have two parts and is related to traversing the levels of biological complexity mentioned before. First, the function of ion channels can be studied to a level of quantitative precision that is often hard to achieve for other proteins. This can be crucial to understanding the link between protein function and cellular processes, particularly in the more interesting cases of disease caused by altered protein function (rather than loss of protein function). Second, ion channels are central to an astounding number of physiological processes, such as nerve and muscle excitation, epithelial transport, and hormone secretion, to name but a few. As the book illustrates, there are many channelopathies in which the molecular defect can be satisfactorily linked to a complex physiological outcome - textbook examples of molecular medicine. These include diseases of blood-pressure regulation caused by mutations in the genes encoding subunits of the epithelial sodium channel, and neurological disorders like startle disease, which is caused by mutations in the gene for a subunit of the glycine-receptor ion channel. Another notable example is the inherited cardiac disorder long QT syndrome, which can be caused by mutations in at least four different ion channel genes.

The book is self-contained in the sense that it provides all the background knowledge necessary to understand the field (three chapters and brief interludes). The author's attention to outlining methodology should be particularly useful to students, as this aspect of how to ask scientific questions is not always taught early enough. The twenty main chapters are devoted to particular families of cloned ion channels. Our knowledge about these groups of channels as well as their apparent medical relevance varies vastly. Ashcroft, however, has turned this variation around and uses it to make the chapters less uniform. All the important information is there, yet the book is in no sense a catalogue (precisely the reason that it makes sense as a book rather than an internet page). It manages to communicate complex ideas with clarity, precision and efficiency, and I personally enjoyed the sense of history that pervades the book, as well as the lucid style in which it is written.
It is almost ironic that, in the face of such successful integration of different levels of biological complexity, the middle ground is somewhat blurry. From the point of cell biology there are wide gaps in our understanding of how ion channels are integrated into cellular pathways and regulative networks. This applies particularly to ion channels that are localized to the membranes of intracellular organelles. Of course, the book cannot reflect this situation, as the author herself recognises in the concluding remark -

"Well, in our country,' said Alice, still panting a little, 'you'd generally get to somewhere else if you ran very fast for a long time as we've been doing.' 'A slow sort of country!' said the Queen. 'Now, here, you see, it takes all the running you can do, to keep in the same place. If you want to get somewhere else, you must run at least twice as fast as that!." Thanks to the headstart Ashcroft provides, the reader should be able to live up to the challenge and 'run twice as fast' to tackle some of the exciting questions raised by Channelopathies.

Blanche Schwappach is at the ZMBH,

Universitate Heidelberg, Im Neuenheimer Feld 282, D-69120, Heidelberg, Germany. e-mail:b.schwappach@zmbh.uni-heidelberg.de

\section{Launch of book section}

In August 2000, Nature Cell Biology launched this new section to provide a forum for the scholarly assessment of newly published books. This section will appear every month and will feature lively, critical reviews covering a broad spectrum of titles ranging from protocols, to in-depth reviews of specific topics, to biographies.

We hope this section will be informative in guiding our readers' decisions whether to buy a particular book, whilst giving the more casual reader an opportunity to become familiar with the state of the field covered by these publications. 